\title{
1 Advanced Multi-Targeted Composite Biomaterial Dressing 2 for Pain and Infection Control in Chronic Leg Ulcers
}

3 Ovidio Catanzano ${ }^{1}$, Rachael Docking ${ }^{2}$, Patricia Schofield ${ }^{2}$, Joshua Boateng $^{1 * \dagger}$

$4 \quad{ }^{1}$ Department of Pharmaceutical, Chemical and Environmental Sciences, Faculty of Engineering 5 and Science, University of Greenwich, Medway, Central Avenue, Chatham Maritime, Kent, UK, 6 ME4 4TB

$7{ }^{2}$ Faculty of Health, Social Care and Education, Anglia Ruskin University, Bishop Hall Lane, 8 Chelmsford, Essex, CM1 1SQ

10 . *Corresponding author: Joshua S. Boateng (J.S.Boateng@greenwich.ac.uk; joshboat40@gmail.com)

11 Ovidio Catanzano (ovid@hotmail.it)

12

13

14 


\section{ABSTRACT}

16 This study aimed to develop advanced biomaterial polysaccharide based dressings to manage

17 pain associated with infected chronic leg ulcers in older adults. Composite carrageenan (CARR)

18 and hyaluronic acid (HA) dressings loaded with lidocaine (LID) and AgNPs were formulated as

19 freeze-dried wafers and functionally characterized for porous microstructure (morphology),

20 mechanical strength, moisture handling properties, swelling, adhesion and lidocaine release.

21 Antimicrobial activity of AgNPs was evaluated (turbidity assay) against Escherichia coli,

22 Pseudomonas aeruginosa and Staphylococcus aureus whilst cell viability studies (MTT) was

23 performed on normal adult human primary epidermal keratinocyte cells. The wafers were soft,

24 flexible and elegant in appearance. HA affected the wafer structure by increasing the resistance

25 to compression but still possessed a balance between toughness and flexibility to withstand

26 normal stresses and prevent damage to newly formed skin tissue respectively. Water uptake was

27 influenced by HA, whilst equilibrium water content and LID release were similar for all the

28 formulations, showing controlled release up to $6 \mathrm{~h}$. AgNPs loaded CARR/HA wafers were

29 effective in inhibiting the growth of both Gram positive and Gram negative bacteria. MTT assay

30 showed evidence that the AgNPs/ LID loaded wafers did not interfere with cell viability and

31 growth. CARR/HA wafers seem to be a promising system to simultaneously deliver LID and

32 AgNPs, directly to infected chronic leg ulcers.

34 KEYWORDS: Antimicrobial activity; Carrageenan/hyaluronate wafers; Chronic leg ulcers;

35 Lidocaine; Silver nanoparticles; Wound healing 


\section{INTRODUCTION}

Pain is very common in leg ulcer patients with about $1 \%$ of the Western population estimated to suffer from chronic leg ulcers (CLUs) (O’Meara et al., 2012) with 17-65\% of these experiencing severe pain (Philips et al., 1994). Leg ulcers are common in older adults due to risk

41 factors including immobility and venous disease (Simon et al., 2004) and affect up to $2 \%$ of the

42 population over 80 years old (Petherick et al., 2013). Pain, together with leaking exudate, odor,

43 restricted mobility and sleep disturbance may be particularly challenging and distressing for

44 patients and have a negative impact on all aspects of daily living, causing depression, anxiety

45 and social isolation (Green et al., 2014). Furthermore, CLUs present a significant socio-

46 economic burden for health systems, with up to $3 \%$ of the entire healthcare budget, spent on

47 managing CLUs (Augustin et al., 2014; Posnett et al., 2008).

48 In CLUs, the wound is stuck in a continuous inflammation cycle, which coupled with

49 local factors (e.g. ischemia, infection and maceration) and underlying pathologies (e.g. diabetic

50 neuropathy, peripheral vascular disease), causes background pain. Pain also results from repeated

51 tissue insults caused by physical trauma, but the most common cause of wound pain is during

52 dressing change, debriding, and wound cleansing. Multilayer compression bandaging has been

53 identified as the gold standard in the treatment of venous leg ulcers (Harding et al., 2015), but

54 exudate control is a critical factor for its success. For this reason, health professionals would

55 welcome a dressing that deals with absorption challenges and help pain management,

56 particularly during dressing changes (Jorgensen et al., 2006).

57 Wound infection contributes to pain by triggering a continuous inflammatory response

58 through the release of inflammatory mediators, stimulating the production of enzymes and free

59 radicals, which can cause tissue damage (White, 2009). The consequent pain-related stress leads 
to reduced immune response to infection and stimulation of pro-inflammatory cytokine

61 production in the wound (Glaser et al., 1999). Therefore, treatment of pain and infection should

62 be equally prioritized (Boateng \& Catanzano, 2015). CLUs are particularly at risk of infection

63 due to high microbial bioburden (White et al., 2006) and the inability of leukocytes for

64 phagocytosis and intracellular killing of microorganisms (Oncul et al., 2007). Different

65 antibiotics have been used against wound infections, but the development of resistance has

66 highlighted the need for alternative solutions. Recently, silver nanoparticles (AgNPs) have been

67 recognized as optimal candidates for overcoming wound infections and due to their broad-

68 spectrum antimicrobial characteristics, have been widely used in CLU (Rizzello \& Pompa,

69 2014). The use of AgNPs-containing dressings in the treatment of infected wounds has been

70 explored as a way to reduce risk of infection in the wound area and avoid delays in wound

71 closure (Boateng et al., 2008; 2015; Hebeisha et al., 2014). However, as noted earlier, wound

72 infection is also a cause of inflammation and pain so the delivery of AgNPs could be an indirect

73 way to reduce wound pain in CLUs.

74 Natural polysaccharides (e.g. chitosan and cellulose) have been reported by several authors as

75 delivery systems for AgNPs for treating infected wounds (El-Naggar et al., 2016; Ganest et al.,

76 2016; Ding et al., 2017; Singla et al., 2017). Carrageenan (CARR) and hyaluronic acid (HA) are

77 two very promising natural polysaccharides for wound dressing application due their good

78 biocompatibility, structural properties and biological activity (Boateng et al., 2013, 2015). CARR

79 is a natural carbohydrate polymer, extracted from intracellular matrix of red seaweeds, widely

80 used in the food industry for its excellent gelling capacity and the three commercially relevant

81 grades are kappa $(\kappa)$, iota (1), and lambda $(\lambda)$. All CARRs are high-molecular-weight

82 polysaccharides made up of repeating galactose units and 3,6 anhydrogalactose (A), joined by 
83 alternating $\alpha-1,3$ and $\beta-1,4$ glycosidic (G) linkages. The main differences between kappa, iota,

84 and lambda CARRs are number and position of the ester sulfate groups on the repeating

85 galactose units and the molecular weight (Cunha \& Grenha, 2016). $\kappa$-CARR has an ester sulfate

86 content of 25 - 30\%, which imparts excellent film and gel forming capacity and together with

87 good biocompatibility, indicate that $\kappa$-CARR could be a versatile biomaterial for drug delivery.

88 Furthermore, $\kappa$-CARR exhibits immune and blood coagulation activities useful in tissue

89 engineering and wound healing (Cunha \& Grenha, 2016; Liu et al., 2015; angestuti \& Kim,

90 2014). During heating CARR first exists as random coil but the chains reorganize themselves as

91 double helices when cooled (Cunha and Grenha, 2015). In general, CARR lends itself to a

92 variety of applications to modify drug release and improve drug dissolution, and recently, our

93 group proposed $\kappa$-CARR based formulations for buccal mucosa drug delivery (Kianfar et al.,

94 2014; Kianfar et al., 2013), and as dressings for drug delivery to wounds (Boateng et al., 2013, 95 2015).

97 units of ( $\beta$ 1-4)-d-glucuronic acid and ( $\beta$ 1-3)-N-acetyl-d-glucosamine found naturally in the

98 body and acts as a structural component of the extracellular matrix (ECM) and mediator of

99 various cellular functions. HA is involved in each phase of wound healing increasing

100 keratinocyte migration and proliferation and facilitating transport of nutrients and waste products

101 (Dicker et al., 2014; Frenkel, 2012). HA was found to accelerate wound healing in both in vitro

102 (Catanzano et al., 2015) and in vivo (Foschi et al., 1990; King et al., 1991) models, and

103 accelerated skin wound healing in patients affected by different chronic wounds (Voigt \& Driver, 104 2012). 
105 The use of local anesthetic in the treatment of chronic wound pain can only give transitory

106 relief in patients that are constantly in pain, whereas the treatment of wound infection alone has

107 no effect on wound acute pain. For these reasons, we hypothesized that a composite

108 polysaccharide dressing comprising CARR and HA, loaded with a local anesthetic and AgNPs

109 could be very beneficial for the patients, acting directly on pain associated with CLUs as well as

110 deal with infection which one of the main causes of chronic inflammatory pain, consequently

111 enhancing wound healing and improving patient well-being. For this purpose, we present an

112 innovative and convenient multi-disciplinary approach based on composite polymeric wafers

113 capable of simultaneous delivery of an anesthetic drug, lidocaine (LID) and AgNPs. Wafers were

114 formulated from gels combining different ratios of CARR and HA, taking advantage of the

115 beneficial and well recognized properties of HA in the wound healing process. Different amounts

116 of LID and AgNPs were tested to evaluate the antimicrobial activity on Gram-negative and

117 Gram-positive bacteria and in vitro cytotoxicity in normal adult human primary epidermal

118 keratinocyte cell lines. 


\section{MATERIALS AND METHODS} 134 this study.

\subsection{Materials}

Low viscosity $\kappa$-carrageenan (Gelcarin GP 812 NF, molecular weight $<100 \mathrm{kDa}, 25 \%$ ester sulfate and 34\% 3,6-AG, stable at $\mathrm{pH}$ above 3.8) was obtained from FMC Biopolymer Corp (Princeton, NY, USA). Sodium hyalorunate Hyasis ${ }^{\circledR}$ (mean molecular weight $0.8 \mathrm{MDa}$, intrinsic viscosity $15 \mathrm{dL} / \mathrm{g}$ ) was a kind gift from Novozyme (Bagsvaerd, Denmark). Silver nanoparticles (AgNPs) was provided as solution in water (10,000 ppm) from Clusternanotech Ltd. (UK). Acetonitrile (HPLC grade), ethanol (laboratory grade), yeast extract, tryptone, 3-(4,5dimethylthiazol-2-yl)-2,5-diphenyltetrazolium bromide (MTT) and streptomycin sulphate (STP) were all obtained from Fisher Scientific (Leicestershire, UK). Lidocaine hydrochloride monohydrate (LID), bovine serum albumin (BSA), and all other chemicals were purchased from Sigma Aldrich (Gillingham, UK). Media, sera, and antibiotics for cell cultures were from ATCC (American Type Culture Collection, USA). Deionized ultra-filtered water was used throughout

\subsection{Preparation of CARR/HA composite wafers}

CARR and CARR/HA wafers were prepared by dispersing the polymers in stirred hot water $\left(70^{\circ} \mathrm{C}\right)$ until completely dissolved to obtain an aqueous polymeric solution $(2 \% \mathrm{w} / \mathrm{v})$ made of only CARR and blends of CARR with HA in different weight ratios $(90 / 10,70 / 30,50 / 50$, labelled as $\mathrm{CARR} / \mathrm{HA}_{10}, \mathrm{CARR} / \mathrm{HA}_{30}$ and $\mathrm{CARR} / \mathrm{HA} \mathrm{A}_{50}$ respectively. For drug-loaded wafers,

LID (10\% w/w based on total polymer weight) and AgNPs (100 - $750 \mu \mathrm{g} /$ wafer $)$ were dissolved directly in the polymeric solution. Solutions were cast in a 24 -well plates $(1 \mathrm{ml}$, well size: $15 \mathrm{~mm}$ diameter, $20 \mathrm{~mm}$ height) and lyophilized using an automated cycle on a Virtis Advantage XL 70 
144 freeze dryer (Biopharma Process Systems, Winchester, UK) to form circular disks. The freeze-

145 drying process involved initially cooling the samples from room temperature to $-50^{\circ} \mathrm{C}(8 \mathrm{~h})$ and

146 heated during the primary drying phase $\left(50 \mathrm{mTorr},-25^{\circ} \mathrm{C}\right)$ for $24 \mathrm{~h}$ to sublimate the ice,

147 followed by $7 \mathrm{~h}$ of secondary drying $\left(10 \mathrm{mTorr}, 20^{\circ} \mathrm{C}\right)$ to remove free water. The wafers were

148 removed from the dish and kept in desiccators over calcium chloride at room temperature till

149 required.

\subsection{Physical and analytical characterization}

The surface morphology of the CARR/HA wafers was analyzed using scanning electron

153 microscopy (SEM). Samples were cut into small, thin pieces, placed on Agar Scientific double-

154 sided carbon adhesive tape on Agar Scientific G301 aluminium stub and coated with gold using

155 Sputter Coater (Edwards 188 Sputter Coater S1508) evaporator for 90 - 120 s. Images were

156 obtained using a Hitachi SU8030 (Hitachi High-Technologies, Krefeld, Germany) scanning

157 microscope under low vacuum at $5.0 \mathrm{kV}$ accelerating voltage.

158 The average porosity of wafers was determined by a fluid replacement method. The

159 geometrical volume $\left(\mathrm{V}_{\mathrm{s}}\right)$ of the wafers $(n=5)$ was calculated by measuring diameter and height,

160 and the pore volume $\left(\mathrm{V}_{\mathrm{p}}\right)$ was measured by ethanol displacement. The dry wafer was weighed

$161\left(\mathrm{~W}_{0}\right)$ and immersed in absolute ethanol at room temperature, and placed in a degasser for 10 min

162 to remove air bubbles. After wiping gently with a filter paper, samples were weighed

163 immediately $\left(\mathrm{W}_{\mathrm{e}}\right)$. The porosity of the wafers was calculated using equation 1:

164

165 Porosity $=\frac{V_{p}}{V_{s}} X 100=\frac{W_{e}-W_{0}}{\rho_{e} V_{S}} \times 100$

[Equation 1] 
167 where $\rho_{\mathrm{e}}$ represents the density of ethanol $\left(0.789 \mathrm{~g} / \mathrm{cm}^{3}\right)$.

168 The apparent density $(\rho)$ of the sponge was calculated using equation 2 :

169

$170 \rho=\frac{W_{0}}{V_{S}}$

[Equation 2]

171

172

For water retention rate, wafer was soaked in water for $20 \mathrm{~min}$, carefully removed, gently

173 wiped on tissue paper to remove the excess water and placed in a centrifuge tube equipped with a

174 mesh bottom, centrifuged at $500 \mathrm{rpm}$ for $2 \mathrm{~min}$ and the wet weight recorded. An average value of

175 five replicates $(n=5)$ for each sample was taken. Water retention rate (WR) was calculated with

176 equation 3 :

177

$178 \quad W R=\frac{M_{h}-M_{d}}{M_{d}} \times 100$

[Equation 3]

180 Where $\mathrm{M}_{\mathrm{h}}$ is the weight $(\mathrm{g})$ of the wafer after centrifugation, and $\mathrm{M}_{\mathrm{d}}$ is the initial dry weight $(\mathrm{g})$.

181 The residual moisture in the wafers was estimated using thermogravimetric analysis

182 (TGA) (Thermal Advantage 2950, TA Instruments, Crawley UK). Samples $(n=4)$ weighing

183 between 3 and $6 \mathrm{mg}$ were placed in a previously tared $70 \mu \mathrm{L}$ aluminium crucible and heated at

$18410^{\circ} \mathrm{C} / \mathrm{min}$ from ambient temperature to $200^{\circ} \mathrm{C}$ under a constant stream of dry nitrogen. The

185 weight loss was plotted against temperature and percentage residual moisture was estimated from

186 the second derivative plot using TA Universal Analysis 2000 software.

187 A Fourier transform infrared spectrometer (FT-IR) equipped with a ZnSe attenuated total

188 reflectance (ATR) crystal accessory (Nicolet 8700 FTIR spectrometer, Thermo Fisher Scientific,

189 Surrey, UK) was used to investigate interaction between CARR and HA during formulation and 
190 presence of unmodified HA. Wafers and starting materials, were placed on the ATR crystal and

191 pressed with a pressure clamp to allow optimal contact between the material and the crystal and

192 FT-IR spectra were collected over $4000-400 \mathrm{~cm}^{-1}$ using a resolution of $1 \mathrm{~cm}^{-1}$.

\section{$193 \quad 2.4 \quad$ Swelling studies}

194 The percentage swelling ratio was determined by placing the wafers in simulated wound fluid 195 (SWF, $\mathrm{CaCl} 0.02 \mathrm{M}, \mathrm{NaCl} 0.4 \mathrm{M}$, Trizma base $0.4 \mathrm{M}$, BSA 2\% w/v). The initial weight of each 196 wafer was recorded and completely immersed in $5 \mathrm{~mL}$ of SWF $\left(37^{\circ} \mathrm{C}\right)$. Samples were carefully 197 taken out, excess SWF removed by carefully blotting with tissue paper, weighed and re-

198 immersed in SWF. Weights were recorded at intervals of $1 \mathrm{~h}$ up to $6 \mathrm{~h}$ and every $24 \mathrm{~h}$ from then

199 onwards until equilibrium was established and SWF replaced after every weight measurement.

200 The percentage swelling ratio (SR \%) was calculated using equation 4:

201

$202 S R \%=\frac{W-W_{0}}{W_{0}} \times 100$

[Equation 4]

203

204 Where $W$ is the mass of the swollen sample and $W_{0}$ is the mass of the initial dry sample. The 205 equilibrium water content (EWC) per cent was calculated using equation 5:

206

$207 \quad \operatorname{EWC}(\%)=\frac{W_{e}-W_{0}}{W_{e}} \times 100$

[Equation 5]

208

209 Where $\mathrm{W}_{\mathrm{e}}$ is the mass of the swollen sample at equilibrium.

210

$211 \quad 2.5 \quad$ Mechanical hardness and in vitro wound adhesive properties 
212 Mechanical properties (resistance to deformation and ease of recovery) of the freeze-dried wafers

213 were investigated by compressing on a Texture Analyzer (TA) (Stable Microsystems Ltd.,

214 Surrey, UK) equipped with $5 \mathrm{~kg}$ load cell and Texture Exponent-32 ${ }^{\circledR}$ software program. A $6 \mathrm{~mm}$

215 cylindrical stainless steel probe was used in compression mode. Wafers $(n=5)$ were compressed

216 at three different locations to a depth of $2 \mathrm{~mm}$ at a speed of $1 \mathrm{~mm} / \mathrm{s}$ using a trigger force of 0.001

$217 \mathrm{~N}$ to determine the effects of HA on the resistance to deformation ('hardness').

218 In vitro wound adhesion was performed using the same apparatus but in tension mode.

219 Samples $(n=5)$ were attached to a $75 \mathrm{~mm}$ diameter probe using double-sided adhesive tape. A

$2206.67 \% \mathrm{w} / \mathrm{v}$ gelatin solution was allowed to set as solid gel in a Petri dish (diameter $88 \mathrm{~mm}$ ) and

$2210.5 \mathrm{~mL}$ of SWF spread over the surface to simulate a moist wound (Boateng et al., 2013, 2015).

222 The probe, lined with wafer, was set to approach the model wound surface with the following

223 conditions: pre-test speed $-0.5 \mathrm{~mm} / \mathrm{s}$; test speed $-0.5 \mathrm{~mm} / \mathrm{s}$; post-test speed $-1.0 \mathrm{~mm} / \mathrm{s}$; applied

224 force - $0.01 \mathrm{~N}$; contact time - $60.0 \mathrm{~s}$; trigger type - auto; trigger force $-0.05 \mathrm{~N}$ and return distance

225 of $10.0 \mathrm{~mm}$ (Pawar et al., 2014). The maximum force required to detach the wafer on the upper

226 probe (stickiness) from the model wound surface, known as the peak adhesive force (PAF), total

227 work of adhesion (WOA) represented by the area under the force versus distance curve, and

228 cohesiveness, defined as the distance travelled by wafer till detached, were calculated using the

229 Texture Exponent-32 ${ }^{\circledR}$ software.

\section{$231 \quad 2.6 \quad$ In vitro $\mathrm{LID}$ release}

232 In vitro release of LID from CARR and CARR/HA wafers $(n=3)$ was evaluated in SWF

233 at $37^{\circ} \mathrm{C}$ under gentle shaking and SWF $(11 \mathrm{~mL})$ was in contact with the wafers only by their

234 lower surface. The SWF was prepared without BSA to avoid blocking of the HPLC column. At 
235 set times, $1.0 \mathrm{~mL}$ of release medium was withdrawn, replaced by the same amount of fresh SWF

236 and analyzed. Concentration of LID within the wafers and amount released at each time point

237 was determined by HPLC using an Agilent 1200 HPLC system (Agilent Technologies, Cheshire,

238 UK) equipped with a Chemstation ${ }^{\circledR}$ software program. The stationary phase was a Gemini C18

239 column (250 mm x $4.6 \mathrm{~mm}, 300 \AA$ A ) (Phenomenex, USA), mobile phase comprised 5\% acetic

240 acid in water $(\mathrm{pH} 3.4)$ and acetonitrile in the ratio of 80:20 (v/v) at flow rate of $1.0 \mathrm{~mL} / \mathrm{min}$,

241 injection volume was $20 \mu \mathrm{L}$ and detection wavelength at $262 \mathrm{~nm}$. The linearity of the response

242 was verified over a LID concentration range of $5-500 \mu \mathrm{g} / \mathrm{ml}\left(\mathrm{R}^{2}=0.997\right)$.

243

$244 \quad 2.7 \quad$ In vitro cytotoxicity

245 Cell viability studies were performed on normal adult human primary epidermal

246 keratinocyte cells (ATCC PCS-200-011). The cells were cultured in cell culture flasks using a

247 complete culture medium consisting of dermal cell basal medium (ATCC ${ }^{\circledR}$ PCS-200-030) plus

248 one keratinocyte growth kit (ATCC ${ }^{\circledR}$ PCS-200-040) containing bovine pituitary extract (BPE), rh

249 TGF $\alpha$, L-glutamine, hydrocortisone hemisuccinate, insulin, epinephrine and apotransferrin and

250 supplemented with $10 \mathrm{IU} / \mathrm{mL}$ of penicillin, and $10 \mu \mathrm{g} / \mathrm{mL}$ of streptomycin. Cultures were

251 maintained in humidified atmosphere of $95 \%$ air and $5 \% \mathrm{CO}_{2}$ at $37^{\circ} \mathrm{C}$. The MTT (3-(4,5-

252 dimethylthiazol-2-yl)-2,5-diphenyltetrazolium bromide) tetrazolium reduction assay was used to

253 evaluate the cell viability when in contact with the wafers (International Standardization

254 Organization, 1992) (details in supplementary information S1).

255

$256 \quad 2.8 \quad$ Antimicrobial assay 
The antimicrobial activity of AgNPs-loaded wafers was tested on E. coli (ATCC 25922),

258 P. aeruginosa (ATCC 10145) and S. aureus (ATCC 29213). Wafers containing $100 \mu \mathrm{g}$

259 (CARR/AgNPs 100$), 250 \mu \mathrm{g}\left(\mathrm{CARR} / \mathrm{AgNPs}_{250}\right), 500 \mu \mathrm{g}\left(\mathrm{CARR} / \mathrm{AgNPs}_{500}\right)$, and $750 \mu \mathrm{g}$

260 (CARR/AgNPs750) each of AgNPs were used. Bacterial suspensions were prepared by taking 2-3

261 fresh colonies grown on Luria Bertani (LB) agar plates and suspended in sterile LB broth with no

262 sodium chloride (Tryptone $10 \mathrm{~g} / \mathrm{L}$, yeast extract $5 \mathrm{~g} / \mathrm{L}$ ). The turbidity was adjusted to 0.5

263 McFarland standard $\left(\sim 10^{8} \mathrm{cfu} / \mathrm{mL}\right)$ and then diluted to $10^{6} \mathrm{cfu} / \mathrm{mL}$ (representing highly infected

264 chronic wounds) with fresh LB broth. Equally weighed samples were placed in sterile plastic

265 tubes, submerged with $10 \mathrm{~mL}$ of bacterial suspension, and incubated at $37^{\circ} \mathrm{C}$ in a shaking

266 incubator (100 rpm/min). Absorbance (A) at $580 \mathrm{~nm}$ of the bacterial suspension incubated with

267 samples $(n=3)$ at different time points $(6,24$ and $48 \mathrm{~h})$ were noted and difference in turbidity

268 was visually analyzed. $400 \mu \mathrm{L}$ of streptomycin sulfate solution $(1 \mathrm{mg} / \mathrm{mL})$, and unloaded wafers

269 were used as positive and negative controls respectively.

270

\section{$271 \quad 2.9 \quad$ Statistical analyses}

272 Statistical analyses were undertaken using GraphPad Prism ${ }^{\circledR}$, version 6.00 (GraphPad Software,

273 La Jolla California USA, www.graphpad.com) and data compared using a Student's t-test and a

274 one-way ANOVA where relevant, with a Bonferroni post-test (parametric methods).

275

\section{RESULTS}

\section{$277 \quad 3.1$ Formulation development}

278 Freeze drying is a simple way to produce porous sponge-like dressings, useful in medium to

279 high exuding wounds (Matthews et al., 2005). Composite CARR/HA wafers possessed a smooth 
280 surface with uniform mass, texture and toughness. All formulations were elegant with no visible

281 cracks but the balance between flexibility and toughness changed depending on HA

282 concentration.

283

\section{$284 \quad 3.2$ Wafer characterization}

285 Representative SEM images of wafers are shown in Figure 1, showing porous

286 interconnecting network of polymeric strands having irregular and hexagonal shaped pores with

287 thin strands. CARR wafers formed an interconnecting network, with an average pore diameter of

$28850 \mu \mathrm{m}$ and an open cell structure with hexagonal shaped pores. Addition of HA resulted in

289 smaller pore sizes with an average pore diameter between 10 and $30 \mu \mathrm{m}$. HA has an important

290 role in porosity development and wafers with higher HA amount showed relatively smaller sized

291 pores and a more irregular matrix structure. Such changes in microstructure can affect other

292 physical properties such as 'hardness' and hydration (Pawar et al., 2014). 

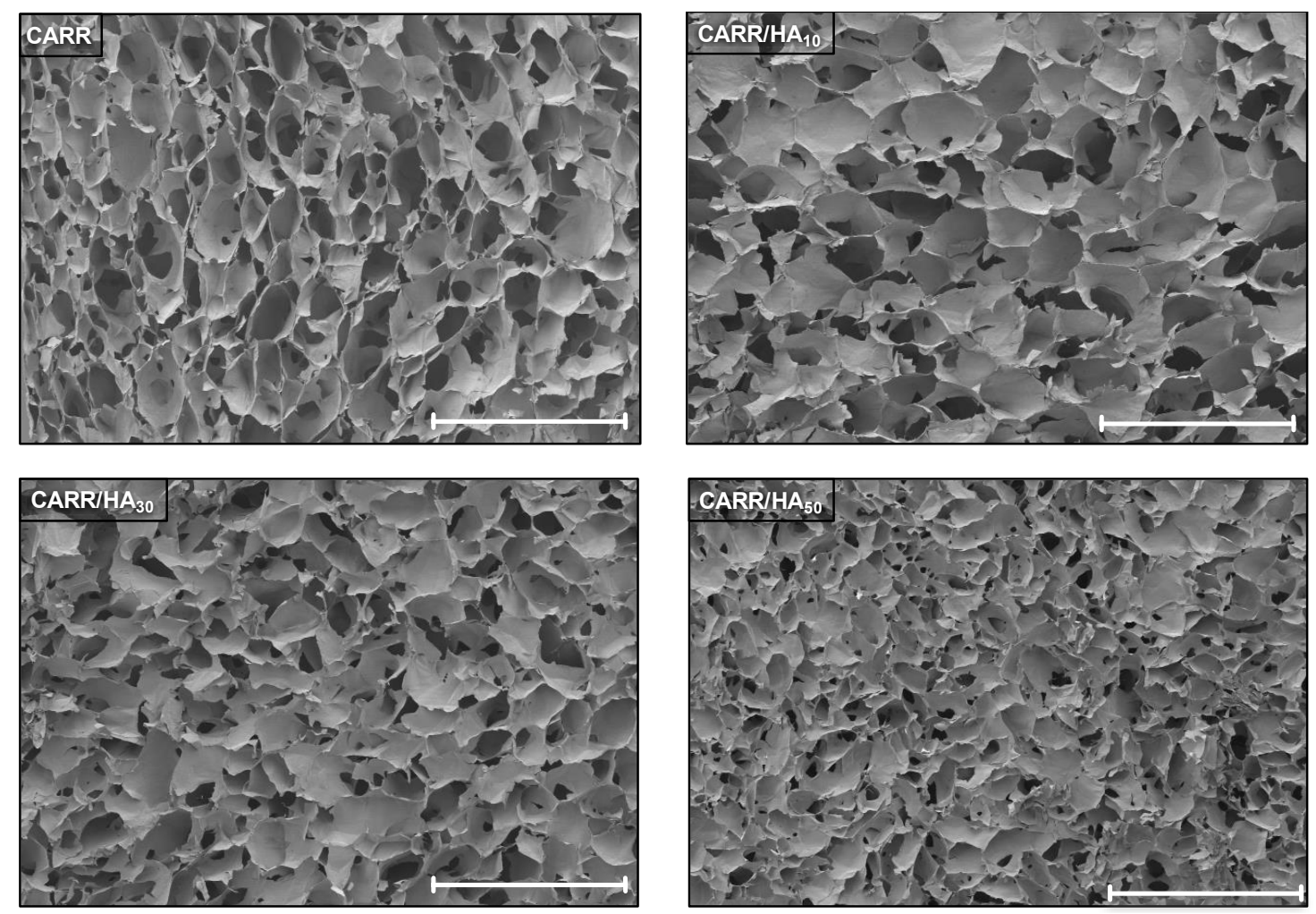

Figure 1: Representative SEM micrographs of CARR and CARR/HA wafers showing differences in porous microstructure with different amounts of HA $(x 40$, scale bar $=1 \mathrm{~mm})$.

The porosity and water handling properties for the various wafers are summarized in

299 Table 1. CARR wafers with more open and ordered pores and solid internal structure, had a

300 water absorption > $3700 \mathrm{~g}$ water per $100 \mathrm{~g}$ of wafer, however addition of HA caused variation in

301 all the physical properties. Both the porosity values, and apparent density of the wafers were

302 influenced by HA, which made them more porous and less dense, therefore decreasing the water

303 absorption. The water retention capacity was highest for CARR/HA 50 and generally higher in

304 CARR/HA wafers compared to CARR wafer, due to the higher hydrophilic character of HA

305 (Tool, 2004) which also resulted in slightly higher residual moisture content for CARR/HA

306 wafers ranging from $17.00 \pm 0.57 \%$ (CARR wafer) to $18.50 \pm 0.28 \%$ (CARR/HA50 wafer).

307 Figure S2 (supplementary data) shows the FTIR spectrum (A) and assignment of the main

308 absorption bands (B) of the raw materials and CARR/HA wafers at different ratios of HA. 
Table 1: Comparison of physical properties between different composite CARR/HA wafers

\begin{tabular}{ccccccc}
\hline & Porosity (\%) & $\begin{array}{c}\text { Apparent } \\
\text { density }\left(\mathbf{m g} / \mathbf{c m}^{\mathbf{3}}\right)\end{array}$ & $\begin{array}{c}\text { Water } \\
\text { absorption (\%) }\end{array}$ & $\begin{array}{c}\text { Water } \\
\text { retention }(\%)\end{array}$ & $\begin{array}{c}\text { EWC (\%) } \\
\text { (\%ontent }(\%)\end{array}$ & $\begin{array}{c}\text { Moisture } \\
\text { cons }\end{array}$ \\
\hline CARR & $92.25 \pm 4.53$ & $26.78 \pm 1.51$ & $3782.7 \pm 86.9$ & $96.91 \pm 0.53$ & $97.40 \pm 0.09$ & $17.00 \pm 0.57$ \\
CARR/HA 50 & $85.00 \pm 2.62$ & $30.32 \pm 1.25$ & $3640.2 \pm 39.1$ & $98.88 \pm 0.57$ & $97.33 \pm 0.03$ & $18.50 \pm 0.28$ \\
CARR/HA 30 & $87.95 \pm 3.55$ & $29.69 \pm 1.75$ & $3394.1 \pm 112.5$ & $97.61 \pm 1.70$ & $97.04 \pm 0.21$ & $17.10 \pm 0.44$ \\
CARR/HA $_{10}$ & $89.06 \pm 2.98$ & $30.55 \pm 0.60$ & $3270.7 \pm 70.8$ & $95.65 \pm 1.33$ & $97.03 \pm 0.08$ & $16.73 \pm 0.29$ \\
\hline
\end{tabular}

\section{$311 \quad 3.3 \quad$ Swelling studies}

312 Figure 2 showed differences in swelling capacity depending on the proportion of HA. The

313 percentage swelling increased with time but the total capacity was lower in the presence of HA

314 which was pronounced after $30 \mathrm{~min}$, where the water uptake was $3528 \pm 61 \%$ and $1966 \pm 27 \%$ for

315 CARR and CARR/HA50 respectively and statistically significant $(p<0.05)$. The curves show that

316 after $6 \mathrm{~h}$ the maximum swelling was achieved for all the formulations except CARR/HA50, which

317 continued to absorb water until $24 \mathrm{~h}$. After 6 h, CARR wafers showed a significantly $(p<0.05)$

318 higher swelling capacity compared to CARR/HA 30 and CARR/HA 50 with a maximum of $3847 \pm$

$31930 \%$, whilst increase in HA caused a decrease in the swelling with CARR/HA 50 showing a 320 maximum value of $2867 \pm 143 \%$. After $6 \mathrm{~h}$ no significant differences are found in swelling between 321 CARR and CARR/HA 10 wafers. 


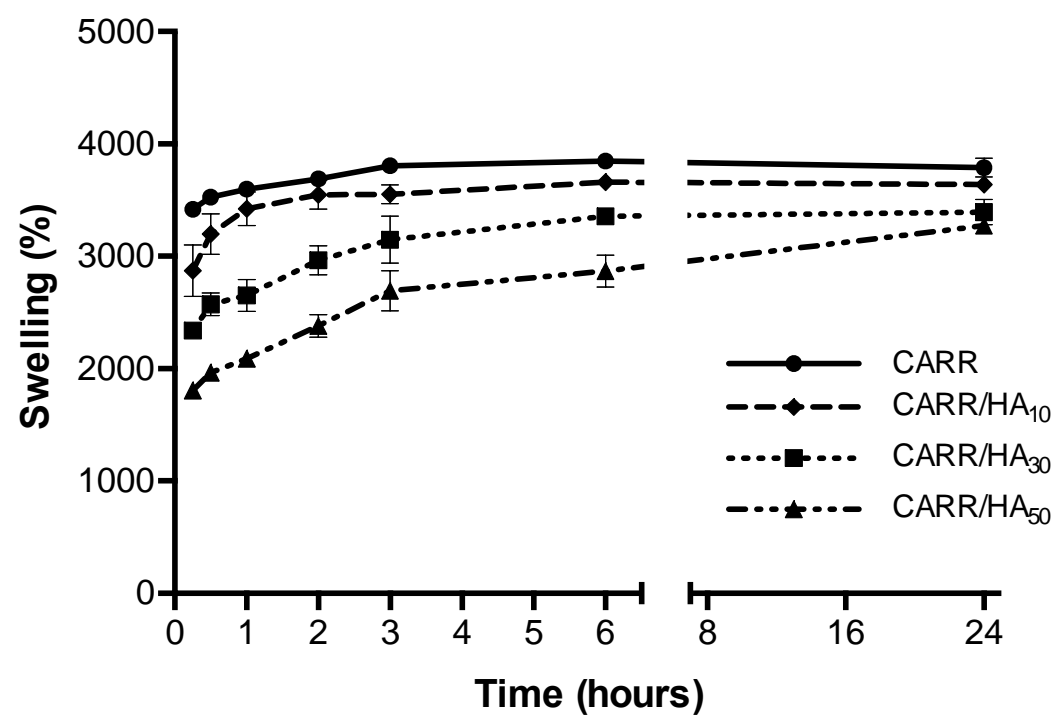

323

324

325

326

327

328 2014).
Figure 2: Swelling profiles of CARR and CARR/HA wafers $(n=3, \pm \mathrm{SD})$. After 6 h CARR wafers have a significantly $(p<0.05)$ higher swelling capacity vs $\mathrm{CARR} / \mathrm{HA}_{30}$ and CARR/HA 50 while the difference between CARR and $\mathrm{CARR} / \mathrm{HA}_{10}$ was not significant. Moreover, no differences were noted in case of LID and AgNPs loaded wafers.

\subsection{Mechanical hardness and in vitro mucoadhesive properties.}

Figure 3A shows the changes in 'hardness' for the wafers with increasing concentration of HA which suggests increased 'hardness' (decreased flexibility), which could affect rate of hydration, swelling and mucoadhesion performance. Figure $3 \mathrm{~B}$ shows the adhesion properties of CARR and composite CARR/HA wafers represented by PAF, WOA and cohesiveness. The CARR wafers had a higher PAF $(0.740 \pm 0.070 \mathrm{~N})$ compared to the CARR/HA50 $(0.355 \pm 0.057 \mathrm{~N})$, with a decreasing trend proportional to the presence of HA. The same trend was observed for WOA and cohesiveness. These three parameters are directly influenced by the physicochemical properties of the wafer such as the pore size distribution and the consequent hydration capacity (Pawar et al., 
A)

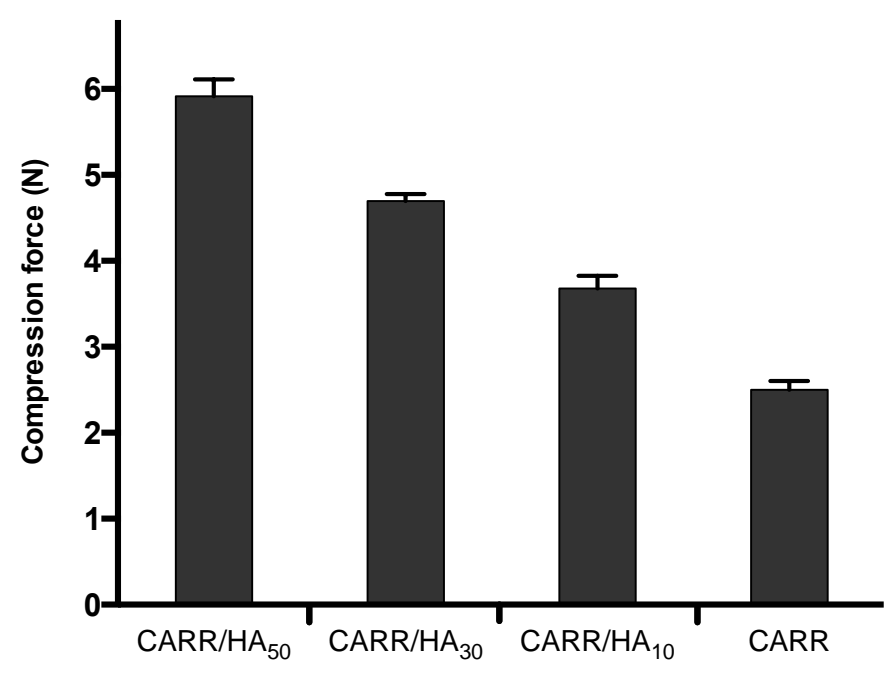

B)

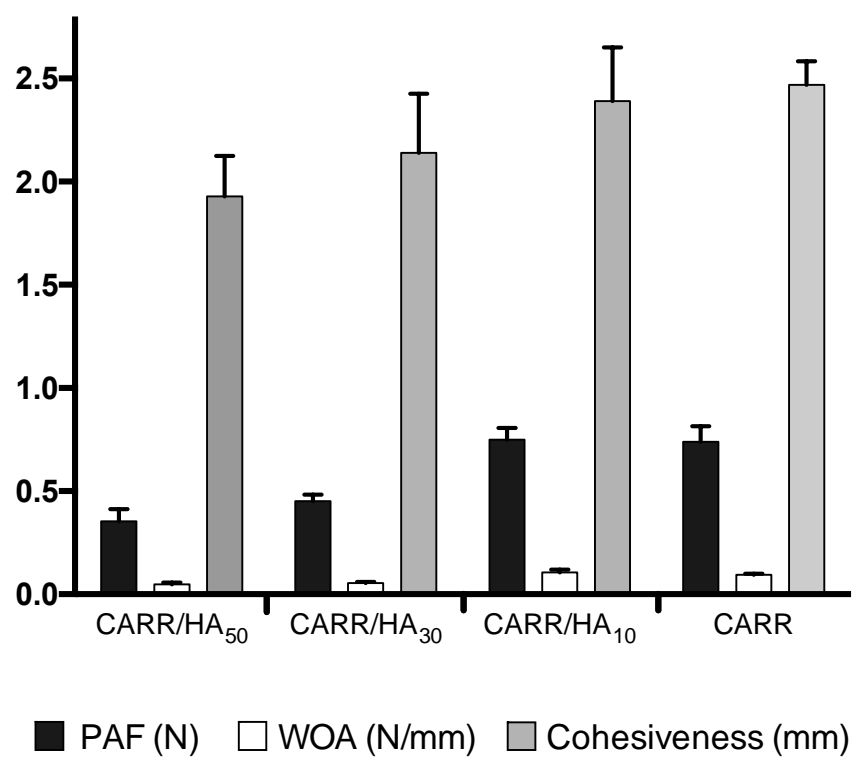
344 Figure 3: A) Resistance to deformation ('hardness') of CARR or CARR/HA wafers compressed at three different $(n=5, \pm \mathrm{SD})$. Ordinate definition for the different parameters was reported in the figure legend. 


\subsection{In vitro $\mathrm{LID}$ release}

The LID release profiles for CARR and CARR/HA wafers $(n=3)$ are shown in Figure 4 ,

350 showing controlled release over $6 \mathrm{~h}$. Wafers with HA appear to release the drug more rapidly

351 initially, though the differences do not appear marked.

352

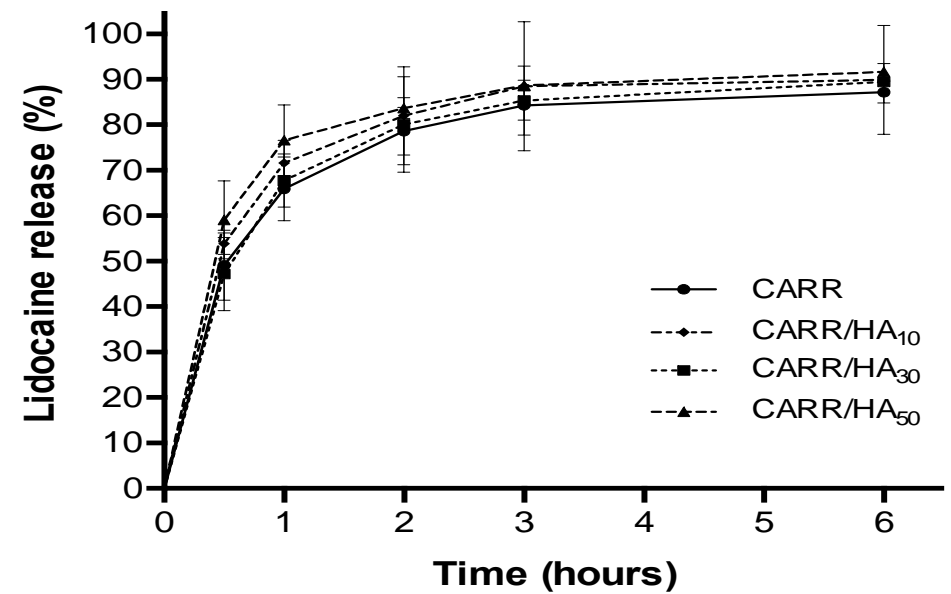

354 Figure 4: In vitro LID cumulative release $(n=3 \pm \mathrm{SD})$ profiles from optimized drug loaded 355 wafers containing different proportions of CARR/HA.

\subsection{In vitro cytotoxicity}

Figure 5 shows the effect of the wafers on keratinocyte cells with all formulations showing

360 a good toxicity profile with a not significant reduction in viability in all the samples if compared

361 with the CARR-BLK wafer. In the range of concentrations studied the presence of both LID and

362 AgNPs seems not to influence the cells viability if compared to the positive control, triton-x, which

363 showed less than $10 \%$ cell viability (data not shown). Though the results show that there is a time

364 dependent mortality at $72 \mathrm{~h}$ below $90 \%$, this is acceptable because the recommended guidelines

365 for in vitro cytotoxicity for medical devices and delivery systems such as wound dressings (DIN 
EN ISO 10993-5) specifies that such materials can be deemed non-cytotoxic for $\geq 70 \%$ cell viability after exposure (Moritz et al., 2014, Cerchiara et al., 2017). Therefore, the results obtained in the current study show that all the formulated dressings are generally safe.

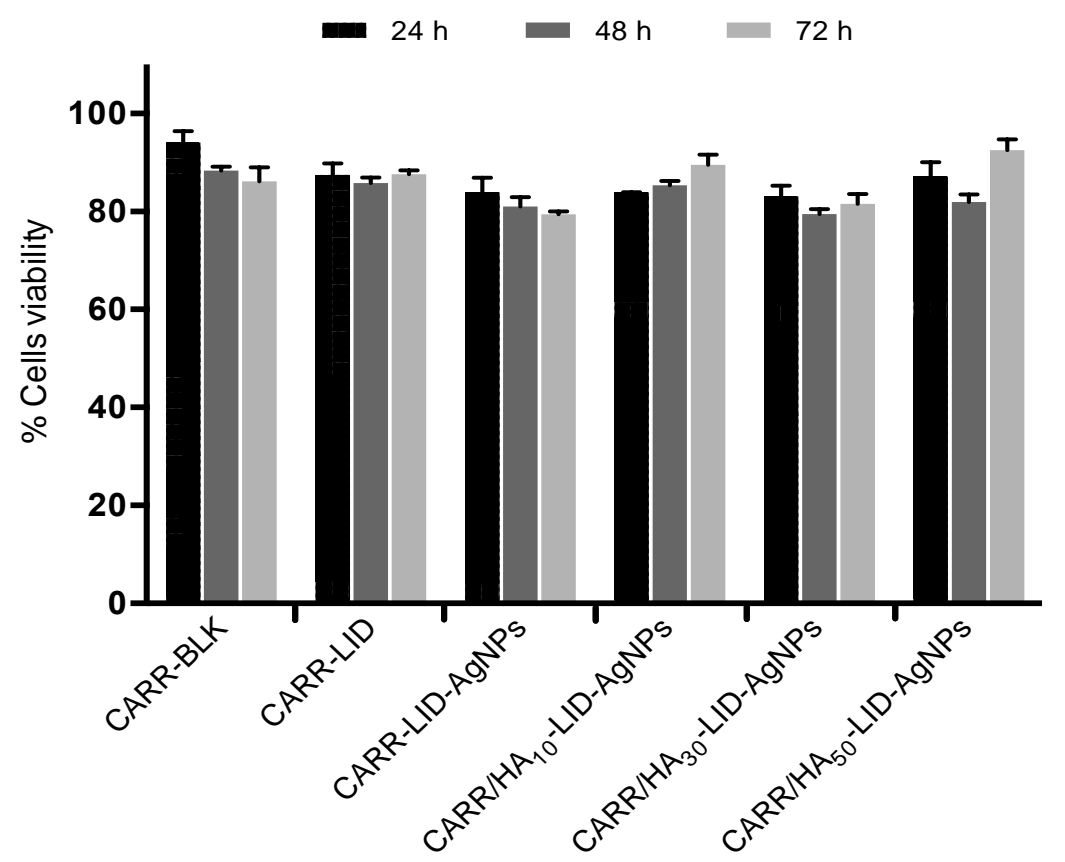

Figure 5. Effect of CARR/HA wafers on cells viability of normal adult human primary epidermal keratinocytes. HaCaT have been seeded at 10,000 cells per well in a 96-well flat-bottomed culture plates and cultured with the extraction media for 24,48 and $72 \mathrm{~h}$. Bars represent the mean \pm standard deviation of three independent experiments $(n=3 \pm \mathrm{SD})$.

\subsection{Antibacterial assays}

Significant difference $\left({ }^{*} p<0.05 ; * * p<0.01\right)$ in the absorbance value at $580 \mathrm{~nm}$ was observed with $S$. aureus, E. coli and P. aeruginosa after $6 \mathrm{~h}$ of incubation for all the samples tested (Figure 6). This activity was strictly related to AgNPs concentration. The activity profile of all the wafers on $P$. aeruginosa was quite different compared to the other bacterial species used in this experiment, since the antimicrobial activity lasted for at least $48 \mathrm{~h}$. Difference in turbidity after 24 $\mathrm{h}$ can be clearly visualized from the photograph of the test tubes containing CARR-AgNPS500 (supplementary data, Figure S4). Furthermore, even though some authors have found that LID has some antibacterial action against E. coli, S. aureus, and P. aeruginosa (Aydin et al., 2001) this 
384 activity was not noticeable in initial trial observations with starting materials which suggested that 385 the LID present in the wafers did not show any recordable antibacterial activity. The wafers were 386 loaded with $10 \%$ LID (w/w based on total polymer weight) which was equivalent to $0.2 \% \mathrm{w} / \mathrm{w}$ 387 and this loading was chosen to be comparable with a high loading commercially available cream. 388 This dose of LID in our wafers was significantly lower than those tested in Aydin et al, (2001), $389(5 \%, 2 \%$ and $1 \%)$ in solution, with only the 5 and $2 \%$ solutions showing broad antibacterial 390 activity. In addition, the LID used in the Aydin et al (2001) study was in solution and therefore in 391 direct immediate contact with the bacteria cells whiles the LID in our wafers will not be released 392 immediately due to the need for initial wafer hydration and subsequent diffusion from the swollen 393 dressing into the medium to allow antibacterial inhibition. Therefore we attributed all the 394 antimicrobial activity to the AgNPs, though future study investigating possible synergistic 395 antibacterial activity of both LID and AgNPs will be interesting, given the potential to reduce 396 possible toxicity by using lower doses of AgNPs. 

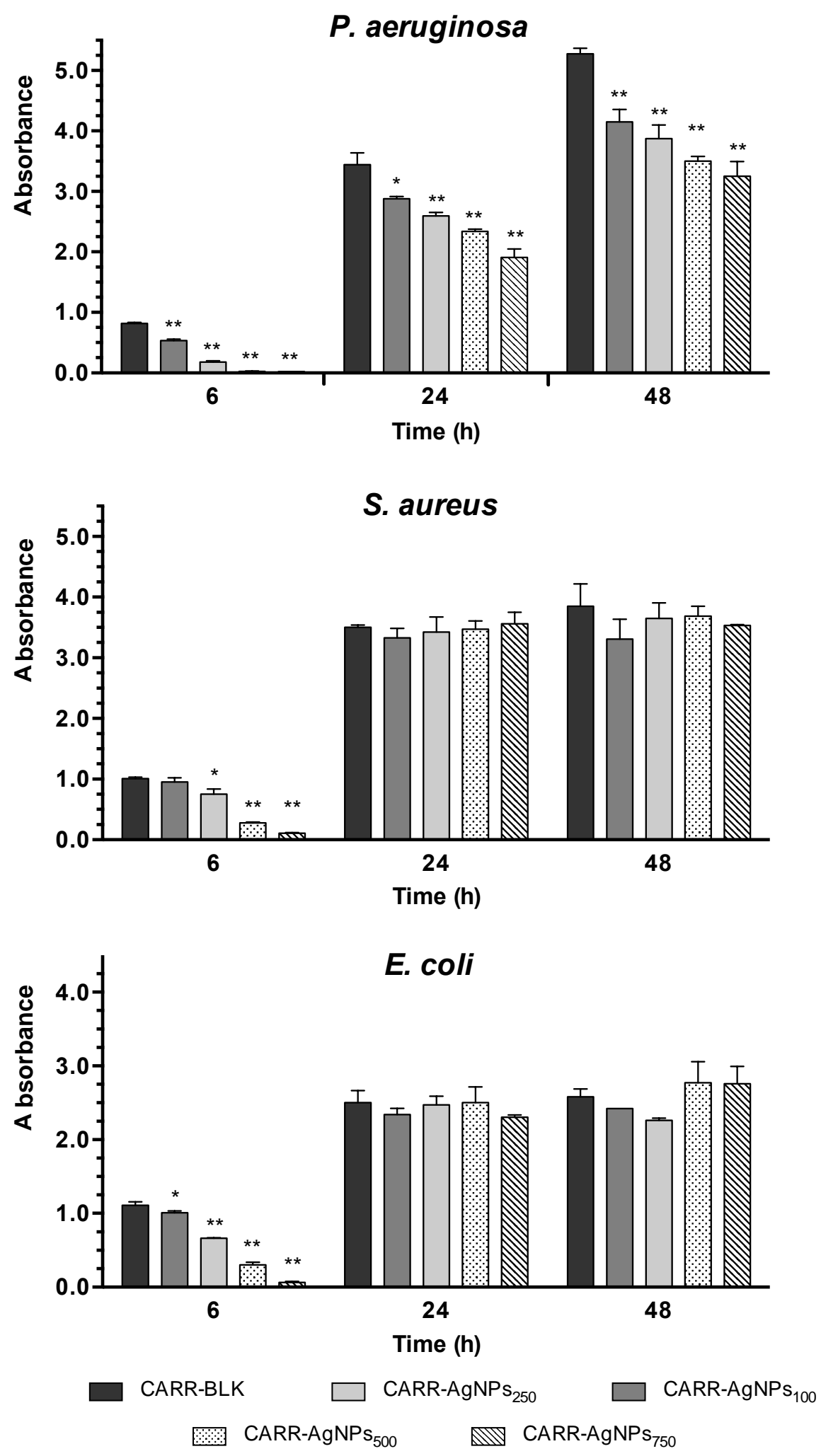

Figure 6: Absorbance values (on ordinate axis) of P. aeruginosa, E. coli S. aureus after 6, 24 and $48 \mathrm{~h}$ of incubation with wafers loaded with different amounts of AgNPs. Significant differences in absorbance in comparison to control are indicated with asterisks $\left(* p<0.05 ;{ }^{* *} p<0.01\right)$. Data are means of three independent experiments $(n=3 \pm \mathrm{SD})$. 


\section{DISCUSSION}

403 Pain management in wound healing represents a challenge for physicians and nurses faced with

404 an increased number of patients affected by different pathologies. Wound dressings are the main

405 devices employed in these therapies and their correct use is an important factor that influences

406 the therapeutic outcome. The simultaneous delivery of a local anesthetic (LID) and an

407 antimicrobial agent (AgNPs) directly to the wound site can be an effective way to respectively

408 target pain itself directly and indirectly on infections, one of the main causes of wound pain due

409 to prolonging the inflammatory phase. Furthermore, the importance of HA in wound repair and

410 tissue regeneration is now considered a critical biomaterial affecting wound healing phases

411 (Frenkel, 2012) and its integration into advanced dressings represents a versatile approach to

412 promote wound healing that can be easily translated into a clinical setting (Catanzano et al., 413 2015).

$414 \quad \kappa$-carrageenan has been employed as films and wafers for wound healing (Boateng et al.,

415 2013; Pawar et al., 2014; Pawar et al., 2013). For our purpose, CARR and composite CARR/HA

416 wafers with different HA content were successfully prepared by freeze-drying. FT-IR

417 spectroscopy was used to confirm the absence of chemical interaction between CARR and HA

418 during the wafer formulation (Figure S2 in supplementary data). No reaction occurred between

419 CARR and HA as there was no significant new peak observed for the composite wafers,

420 suggesting that the two polymers were readily compatible within the composite wafer matrix.

421 The characteristic peak of pure HA appears almost at the same position in the FT-IR spectra of

422 CARR/HA wafers, despite the changes in peak intensity attributed to different amounts of HA

423 used. From a microscopic point of view, all the wafers were porous with an interconnecting

424 network, but micrographs from the SEM shows the effect of HA on the pore shape. CARR/HA 
425 wafers pores are smaller with reduced depth since the HA partially fills the gaps between the

426 CARR chains, which also explains the reduction in porosity. This effect of HA on porosity was

427 also confirmed by evaluating the wafer recovery after deformation at different compression

428 depths (Supplementary data, Figure S3).

429 During wound dressing formulation, pore size and depth should be optimized as they

430 could significantly affect dressing performance in terms of hydration capacity, adhesion,

431 swelling and drug release (Kianfar et al., 2014). Furthermore, a uniform and solid porous

432 structure of crosslinked sponges should "lock" the water molecules and prevent easy runoff of

433 exudate. Attaining a dry state after lyophilization is essential to reduce molecular mobility and

434 hence, increased shelf life. Since no product melt-back was observed, the robustness of the

435 freeze-drying cycle is depicted, with the primary drying stage removing all the loose water. The

436 residual moisture content was derived from the loss in weight and represent bound water that

437 remains after the freeze-drying process. The swelling capacity is an essential characteristic, as

438 CLUs generally produce significant amounts of exudate that should be quickly absorbed and

439 retained over a prolonged period. The ability to absorb fluids and retain moisture without leaking

440 is essential for application on suppurating wounds, where high water retention capacity is

441 required (Boateng et al., 2008). During wound healing, moisture reactive dressings are able to

442 maintain a moist wound environment whilst at the same time avoiding the accumulation of

443 excess exudate on the wound, which can slow down wound healing and cause skin maceration

444 (Boateng \& Catanzano, 2015). The water absorption kinetics of the wafers developed in this

445 study was mainly related to the composition of HA, despite the hydrophilic and polyanionic

446 characteristics of both polymers. This behavior was recently reported (Catanzano et al., 2017)

447 and can be attributed to the HA chain stearic hindrance and increased internal micro-viscosity 
within the cross-linked network that opposes the osmotic force, which drives water molecules

449 into the matrix. This is interesting, as the rate and duration of swelling, determines the ability of

450 the dressing to control drug release over a prolonged period, without the need for regular

451 dressing change (King et al., 1991).

452 The ideal dressing is required to be tough to allow ease of application without breaking

453 but should be soft and flexible to avoid possible contact irritation on the wound and the

454 destruction of newly formed skin tissue (Boateng et al., 2010). The increase in 'hardness' with

455 the increasing HA could affect swelling and mucoadhesion performance as 'hardness' is

456 indicative of wafer density in the internal matrix which affects the rate of water ingress,

457 hydration, impacting on swelling and adhesion (King et al., 1991; Boateng et al., 2010).

458 The use of local anesthetics in wound management is controversial due to their reported

459 delaying effects on wound healing mainly attributable to a reduction in collagen synthesis

460 (Chvapil et al., 1979). However, as demonstrated by Drucked et al., local infiltration of LID does

461 not substantially alter wound healing as they observed no differences in the morphology and

462 mechanical properties of the wounds (Drucker et al., 1998). Furthermore, LID has a lower

463 incidence of allergic reactions than the ester-type anesthetics such as procaine and tetracaine

464 (Popescu \& Salcido, 2004). Though solid lipid microparticles (Albertini et al., 2013) or

465 electrospun scaffolds (Thakur et al., 2008) have been proposed for controlled delivery of LID to

466 the wound site, they are difficult for large scale production and in clinical settings. The purpose

467 of this work was the development of a simple, economical but effective advanced multi-

468 functional composite dressing with LID directly dispersed in the polymeric matrix. Drug release

469 from biodegradable polymeric matrices is strictly correlated to water ingress into the device $(\mathrm{Fu}$

$470 \&$ Kao, 2010). For wound dressings, drug release is driven by the exudate first hydrating the 
471 polymer to cause swelling, dissolving and releasing the drug by gradual diffusion through the

472 swollen gel to the wound site. HA caused a significant $(p<0.05)$ decrease in water uptake but

473 had minimal influence on LID release. The drug release was sustained up to $6 \mathrm{~h}$ in all the

474 formulations, which implies that the drug release is mainly determined by the CARR, however

475 HA is still a vital dressing component, given its known wound healing properties. Furthermore,

476 enough LID was released from all the formulations (about 50\%) in the first hour of dissolution

477 study. This suggests that the dressing could potentially manage pain within the first 60 minutes

478 of application which is important to quickly relieve the severe acute pain, common in leg ulcers

479 especially during dressing change, wound cleansing and new dressing application. However,

480 further in vivo studies are needed to confirm the real pain relieving and wound healing activities

481 of these dressings

482 Keratinocytes are the major cellular component of the epidermis and are directly involved

483 in re-epithelialization (Pastar et al., 2014) and provide a reliable in vitro model of re-

484 epithelialization phase in wound healing (Sivamani et al., 2007). For the toxicity studies the

485 higher concentration of AgNPs $(750 \mu \mathrm{g} /$ wafer $)$ was chosen among all the formulations prepared

486 and the keratinocyte viability was comparable to that achieved with the blank wafer. All

487 formulations showed a similar viability profile and the addition of both LID and AgNPs in the

488 range of concentrations studied did not have any effect on cell viability. On the basis of toxicity

489 data, the AgNPs maximum amount in the hydrogels was fixed at $750 \mu \mathrm{g} / \mathrm{wafer}$ in all the

490 subsequent experiments.

491 Wound colonization by bacteria is common in chronic wounds and in some cases, low

492 levels of bacteria seems to be beneficial to the wound healing process (Edwards \& Harding,

493 2004). The progression from colonization to infection depends on the bacterial count, host 
494 immune response, number of different species present, virulence of the organisms and

495 synergistic interactions between the different species (Edwards \& Harding, 2004). AgNPs are

496 well established as antibacterial and dependent on physicochemical properties of the

497 nanoparticles, particularly the surface characteristics and particle size. For this study, very small

498 AgNPs with a narrow size distribution (mean diameter $0.8 \mathrm{~nm}$ ) and a low polydispersity index

499 (0.110) were used. Several studies have reported the size dependent activity of AgNPs (Rizello

500 \& Pompa, 2014) with small AgNPs (<20 nm) inducing stronger bactericidal effect compared to

501 larger ones due to larger area-to-volume ratio and higher total surface area for membrane

502 interaction. The antimicrobial properties of AgNPs loaded wafers were tested on the most

503 common microorganisms present in CLUs (Ramani et al., 1991), and as expected the

504 antibacterial activity was strictly related to AgNPs concentration. The AgNP loaded wafers were

505 able to kill all the bacteria tested after $6 \mathrm{~h}$ of contact time while a different antimicrobial activity

506 was detected between Gram-positive and Gram-negative bacteria after 24 and $48 \mathrm{~h}$, possibly due

507 the structural differences between the two classes. However, the Gram-negative E. coli showed a

508 lower susceptibility after 24 and $48 \mathrm{~h}$ to CARR-AgNPs wafers than the other Gram-negative

509 strain (P. aeruginosa). Apart from the composition of the outer cell layers, AgNPs antibacterial

510 activity may also be related to the features of the individual bacterial species, and some strain-

511 specific variation in MICs and MBCs was observed for E. coli (Ruparelia et al., 2008).

\section{CONCLUSIONS}

514 In the current study, we hypothesized that composite CARR/HA based wafers, loaded with LID 515 and AgNPs could potentially address pain associated with CLUs as well as deal with infection 516 which is one of the main causes of chronic inflammatory pain and this was confirmed from the 
517 results. We have demonstrated how lyophilized wafers can be designed to obtain a simple,

518 economical but effective multi-targeted composite wound dressing useful for pain management

519 in CLUs. CARR/HA wafers with various HA contents showed a porous nature with an

520 interconnecting network and pore size related to the amount of HA which was also confirmed by

521 texture analysis and swelling studies. Texture analysis also confirmed the good handling

522 properties that together with ease of preparation are essential to provide a rapid and effective

523 treatment for CLUs in hospitalized patients. The fast drug release and the effective antimicrobial

524 activity confirm that the association of an analgesic drug (LID) with an antimicrobial compound

525 (AgNPs) could further increase the effectiveness of this dressing in pain and infection

526 management. Finally, CARR/HA wafers seems to be a very promising system for the treatment

527 of wound pain, however, further studies are needed to evaluate the in vivo wound healing

528 activities of the dressings. 


\section{REFERENCES}

530 Albertini, B., Di Sabatino, M., Calonghi, N., Rodriguez, L., Passerini, N. (2013). Novel

531 multifunctional platforms for potential treatment of cutaneous wounds: development and in vitro 532 characterization. International Journal of Pharmaceutics, 440, 238-249.

533 Augustin, M., Brocatti, L.K., Rustenbach, S.J., Schafer, I., Herberger, K. (2014). Cost-of-illness 534 of leg ulcers in the community. International Wound Journal, 11, 283-292.

535 Aydin, O.N., Eyigor, M.; Aydin, N. Antimicrobial activity of ropivacaine and other local 536 anaesthetics, European Journal of Anaesthesiology 2001, 18, 687-694.

537 Boateng, J.S., Pawar, H.V., Tetteh, J. (2013). Polyox and carrageenan based composite film 538 dressing containing anti-microbial and anti-inflammatory drugs for effective wound healing, 539 International Journal of Pharmaceutics, 441, 181-191.

540 Boateng, J.S., Pawar, H.V., Tetteh, J. (2015).Evaluation of in vitro wound adhesion 541 characteristics of composite film and wafer based dressings using texture analysis and FTIR 542 spectroscopy: a chemometrics factor analysis approach. RSC Advances, 5, 107064-107075.

543 Boateng, J.S., Matthews, K.H., Stevens, H.N., Eccleston, G.M. (2008).Wound healing dressings 544 and drug delivery systems: a review. Journal of Pharmaceutical Sciences, 97, 2892-2923.

545 Boateng, J.S., Catanzano, O. (2015). Advanced Therapeutic Dressings for Effective Wound 546 Healing-A Review. Journal of Pharmaceutical Sciences, 104, 3653-3680.

547 Boateng, J.S., Auffret, A.D., Matthews, K.H., Humphrey, M.J., Stevens, H.N., Eccleston, G.M. 548 (2010). Characterisation of freeze-dried wafers and solvent evaporated films as potential drug 549 delivery systems to mucosal surfaces. International Journal of Pharmaceutics, 389, 24-31.

550 Catanzano, O., D'Esposito, V., Acierno, S., Ambrosio, M.R., De Caro, C., Avagliano, C., Russo, 551 P., Russo, R., Miro, A., Ungaro, F., Calignano, A., Formisano, P., Quaglia, F. (2015). Alginate552 hyaluronan composite hydrogels accelerate wound healing process. Carbohydrate Polymers, $553 \quad 131,407-414$.

554 Catanzano, O., D'Esposito, V., Pulcrano, G., Maiolino, S., Ambrosio, M.R., Esposito, M., Miro, 555 A., Ungaro, F., Formisano, P., Catania, M.R., Quaglia, F. (2017). Ultra-small silver nanoparticles 
556 embedded in alginate-hyaluronic acid hybrid hydrogels for treating infected wound.,

557 International Journal of Polymer Materials: Polymer Biomaterials (In press).

558 Cerchiara, T., Abruzzo, A., Palomino, R.A.N.H., Vitali, B., De Rose, R., Chidichimo, G., 559 Ceseracciu, L., Athanassiou, A., Saladini, B. (2017). Spanish Broom (Spartium junceum L.) fibers 560 impregnated with vancomycin-loaded chitosan nanoparticles as new antibacterial wound dressing: 561 Preparation, characterization and antibacterial activity. European Journal of Pharmaceutical 562 Sciences, 99, 2017, 105-112.

563 Chvapil, M., Hameroff, S.R., O'Dea, K., Peacock, Jr E.E. (1979). Local anesthetics and wound 564 healing. Journal of Surgery Researcy, 27, 367-371.

565 Cunha, L., Grenha, A. (2016). Sulfated seaweed polysaccharides as multifunctional materials in 566 drug delivery applications. Marine Drugs, 14, 42, DOI:10.3390/md14030042

567 Dicker, K.T., Gurski, L.A., Pradhan-Bhatt, S., Witt, R.L., Farach-Carson, M.C., Jia, X. (2014). 568 Hyaluronan: a simple polysaccharide with diverse biological functions, Acta Biomaterialia, 10, 569 1558-1570.

570 Ding, L., Shan, X., Zhao, X., Zha, H., Chen, X., Wang, J., Cai, C., Wang, X., Li, G., Jiejie Hao, 571 J., Yu, G. (2017). Spongy bilayer dressing composed of chitosan-Ag nanoparticles and chitosan572 Bletilla striata polysaccharide for wound healing applications. Carbohydrate Polymers, 157, 573 1538-1547.

574 Drucker, M., Cardenas, E., Arizti, P., Valenzuela, A., Gamboa, A. (1998). Experimental studies 575 on the effect of lidocaine on wound healing. World Journal of Surgery, 22, 394-398.

576 Edwards, R., Harding, K.G. (2004). Bacteria and wound healing. Current Opinion in Infectious 577 Diseases, 17, 91-96.

578 El-Naggar M.E., Abdelgawad A.M., Salas C., Rojas O.J. (2016). Curdlan in fibers as carriers of 579 tetracycline hydrochloride: Controlled release and antibacterial activity, Carbohydrate Polymers $580154,194-203$.

581 Foschi, D., Castoldi, L., Radaelli, E., Abelli, P., Calderini, G., Rastrelli, A., Mariscotti, C., 582 Marazzi, M., Trabucchi, E. (1990). Hyaluronic acid prevents oxygen free-radical damage to 583 granulation tissue: a study in rats. International Journal of Tissue Reactivity, 12, 333-339. 
584 Frenkel, J.S. (2012). The role of hyaluronan in wound healing. International Wound Journal,11, $585 \quad 159-163$.

586 Fu, Y., Kao, W.J. (2010). Drug release kinetics and transport mechanisms of non-degradable and 587 degradable polymeric delivery systems, Expert Opinion on Drug Delivery, 7, 429-444.

588 Ganesh, M., Aziz, A.S., Ubaidulla, U., Hemalatha, P., Saravanakumar, A., Ravikumar, R., Peng, 589 M.M., Choi, E.Y., Jang, H.T. (2016). Sulfanilamide and silver nanoparticles-loaded polyvinyl 590 alcohol-chitosan composite electrospun nanofibers: Synthesis and evaluation on synergism in 591 wound healing. Journal of Industrial and Engineering Chemistry; 39, 127-135.

592 Glaser, R., Kiecolt-Glaser, P.K., Marucha, P.T., MacCallum, R.C., Laskowski, B.F., Malarkey, 593 W.B. (1999). Stress-related changes in proinflammatory cytokine production in wounds.

594 Archives of Genereal Psychiatry, 56, 450-456.

595 Green, J., Jester, R., McKinley, R., Pooler, A. (2014). The impact of chronic venous leg ulcers: a 596 systematic review. Journal of Wound Care, 23, 601-612.

597 Harding, K., Dowsett, C., Fias, L., Jelnes, R., Mosti, G., Öien, R., Partsch, H., Reeder, S., Senet, 598 P., Soriano, J.V., Vanscheidt, W. (2015). Simplifying venous leg ulcer management: Consensus 599 recommendations. Wounds International, (Available at

600 http://www.woundsinternational.com/consensus-documents/view/simplifying-venous-leg-ulcer601 management).

602 Hebeisha A., El-Rafiea M.H., EL-Sheikha M.A., Seleemb A.A., El-Naggara M.E. (2014).

603 Antimicrobial wound dressing and anti-inflammatory efficacy of silver nanoparticles, 604 International Journal of Biological Macromolecules, 65, 509-515.

605 International Standardization Organisation, ISO 10993-5. (1992). Biological Evaluation of 606 Medical Devices, Part 5: Tests for Cytotoxicity, in Vitro Methods, Geneva.

607 Jorgensen, B., Friis, G.J., Gottrup F. (2006). Pain and quality of life for patients with venous leg 608 ulcers: Proof of concept of the efficacy of Biatain-Ibu, a new pain reducing wound dressing.

609 Wound Repair and Regeneration, 14(3), 233-239.

610 Kianfar, F., Antonijevic, M., Chowdhry, B., Boateng, J.S. (2013). Lyophilized wafers 611 comprising carrageenan and pluronic acid for buccal drug delivery using model soluble and 612 insoluble drugs, Colloids Surfaces B: Biointerfaces, 103, 99-106. 
613 Kianfar, F., Ayensu, I., Boateng, J.S. (2014). Development and physico-mechanical

614 characterization of carrageenan and poloxamer-based lyophilized matrix as a potential buccal

615 drug delivery system, Drug Development and Industrial Pharmacy, 40, 361-369.

616 King, S.R., Hickerson, W.L., Proctor, K.G. (1991). Beneficial actions of exogenous hyaluronic

617 acid on wound healing. Surgery, 109, 76-84.

618 Liu, J., Zhan, X., Wan, J., Wang, Y., Wang, C. (2015). Review for carrageenan-based

619 pharmaceutical biomaterials: favourable physical features versus adverse biological effects.

620 Carbohydrate Polymers, 121, 27-36.

621 Matthews, K.H., Stevens, H.N.E., Auffret, A.D., Humphrey, M.J., Eccleston, G.M. (2005).

622 Lyophilised wafers as a drug delivery system for wound healing containing methylcellulose as a 623 viscosity modifier. International Journal of Pharmaceutics, 289, 51-62.

624 Moritz, S., Wiegand, C., Wesarg, F., Hessler, N., Muller, F.A., Kralisch, D., Hipler, U.C., Fischer, 625 D. (2014). Active wound dressing based on bacterial nanocellulose as drug delivery system for 626 octenidine. International Journal of Pharmaceutics, 471, 45-55.

627 O'Meara, S., Cullum, N., Nelson, E.A., Dumville, J.C. (2012). Compression for venous leg 628 ulcers. Cochrane Database Systematic Reviews, 11, CD000265.

629 Oncul, O., Yildiz, S., Gurer, U.S., Yeniiz, E., Qyrdedi, T., Top, C., Gocer, P., Akarsu, B., 630 Cevikbas, A., Cavuslu, S. Effect of the function of polymorphonuclear leukocytes and 631 interleukin-1 beta on wound healing in patients with diabetic foot infections. The Journal of 632 Infection, 54, 250-256.

633 Pangestuti, R., Kim, S.K. (2014). Biological activities of carrageenan, Advanced Food Nutrition 634 Research, 72, 113-124.

635 Pastar, I., Stojadinovic, O., Yin, N.C., Ramirez, H., Nusbaum, A.G., Sawaya, A., Patel, S.B., 636 Khalid, L., Isseroff, R.R., Tomic-Canic, M. (2014). Epithelialization in Wound Healing: A 637 Comprehensive Review. Advanced Wound Care (New Rochelle), 3, 445-464.

638 Pawar, H.V., Tetteh, J., Boateng, J.S. (2013). Preparation, optimisation and characterisation of 639 novel wound healing film dressings loaded with streptomycin and diclofenac. Colloids and 640 Surfaces B: Biointerfaces, 102, 102-110. 
641 Pawar, H.V., Boateng, J.S., Ayensu, I., Tetteh, J. (2014). Multifunctional medicated lyophilised

642 wafer dressing for effective chronic wound healing. Journal of Pharmaceutical Sciences, 103,

$643 \quad 1720-1733$.

644 Petherick, E.S., Cullum, N.A., Pickett, K.E. (2013). Investigation of the effect of deprivation on 645 the burden and management of venous leg ulcers: a cohort study using the THIN database, PLoS 646 One, 8, e58948

647 Phillips, T., Stanton, B., Provan, A., Lew, R. (1994). A study of the impact of leg ulcers on 648 quality of life: financial, social, and psychologic implications. Journal of American Academy of 649 Dermatology, 31, 49-53.

650 Popescu, A., Salcido, R.S. (2004). Wound pain: a challenge for the patient and the wound care 651 specialist. Advanced Skin Wound Care, 17, 14-20.

652 Posnett, J., Franks, P.J. (2008). The burden of chronic wounds in the UK, Nursing Times, 104, 653 44-45.

654 Ramani, A., Ramani, R., Shivananda, P.G., Kundaje, G.N. (1991). Bacteriology of diabetic foot 655 ulcers, Indian Journal of Pathology and Microbiology, 34, 81-87.

656 Rizzello, L., Pompa, P.P. (2014). Nanosilver-based antibacterial drugs and devices:

657 mechanisms, methodological drawbacks, and guidelines. Chemical Society Reviews, 43, 15016581518.

659 Ruparelia, J.P., Chatterjee, A.K., Duttagupta, S.P., Mukherji, S. (2008). Strain specificity in 660 antimicrobial activity of silver and copper nanoparticles. Acta Biomaterialia, 4, 707-716.

661 Simon, D.A., Dix, F.P., McCollum, C.N. (2004). Management of venous leg ulcers, British 662 Medical Journal, 328, 1358-1362.

663 Singla, R., Soni, S., Kulurkar, P.M., Kumari, A., Mahesh S., Patial, V., Padwad, Y.S., Yadav, S.K. 664 (2017). In situ functionalized nanobiocomposites dressings of bamboo cellulose nanocrystals and 665 silver nanoparticles for accelerated wound healing. Carbohydrate Polymers, 155, 152-162.

666 Sivamani, R.K., Garcia, M.S., Isseroff, R.R. (2007). Wound re-epithelialization: modulating 667 keratinocyte migration in wound healing. Frontiers Bioscience: A Journal and Virtual Library, $668 \quad 12,2849-2868$. 
669 Thakur, R.A., Florek, C.A., Kohn, J., Michniak, B.B. (2008). Electrospun nanofibrous polymeric 670 scaffold with targeted drug release profiles for potential application as wound dressing.

671 International Journal of Pharmaceutics, 364, 87-93.

672 Toole, B.P. (2004). Hyaluronan: From extracellular glue to pericellular cue, Nature Reviews in 673 Cancer, 4, 528-539.

674 Voigt, J., Driver, V.R. (2012). Hyaluronic acid derivatives and their healing effect on burns, 675 epithelial surgical wounds, and chronic wounds: a systematic review and meta-analysis of 676 randomized controlled trials. Wound Repair and Regeneration, 20, 317-331.

677 White, R.J. (2009). Wound infection-associated pain. Journal of Wound Care, 18, 245-249.

678 White, R.J., Cutting, K., Kingsley, A. (2006). Topical antimicrobials in the control of wound 679 bioburden, Ostomy/Wound Management, 52, 26-58.

680 